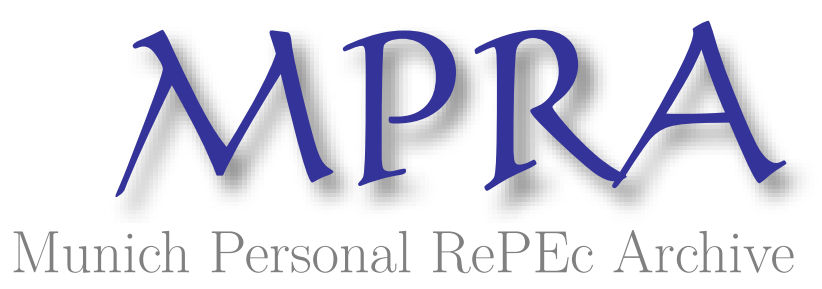

\title{
Innovation creation and diffusion in a social network: an agent based approach
}

Lamieri, Marco and Ietri, Daniele

27 April 2004

Online at https://mpra.ub.uni-muenchen.de/445/

MPRA Paper No. 445, posted 13 Oct 2006 UTC 


\title{
Innovation creation and diffusion in a social network: an agent based approach
}

\author{
Daniele Ietri and Marco Lamieri*
}

October 13, 2006

\begin{abstract}
Market is not only the result of the behaviour of agents, as we can find other forms of contact and communication. Many of them are determined by proximity conditions in some kind of space: in this paper we pay a particular attention to relational space, that is the space determined by the relationships between individuals.

The paper starts from a brief account on theoretical and empirical literature on social networks. Social networks represent people and their relationships as networks, in which individuals are nodes and the relationships between them are ties. In particular, graph theory is used in literature in order to demonstrate some properties of social networks summarised in the concept of "Small Worlds". The concept may be used to explain how some phenomena involving relations among agents have effects on multiple different geographical scales, involving both the local and the global scale.

The empirical section of the paper is introduced by a brief summary of simulation techniques in social science and economics as a way to investigate complexity. The model investigates the dynamics of a population of firms (potential innovators) and consumers interacting in a space defined as a social network. Consumers are represented in the model in order to create a competitive environment pushing enterprises into innovative process (we refer to Schumpeter's definition): from interaction between consumers and firms innovation emerges as a relational good.
\end{abstract}

* "Dipartimento Interateneo Territorio, Politecnico e Università di Torino" (ietri@econ.unito.it) and "Dipartimento di matematica e statistica applicata alle scienze umane Diego De Castro, Università degli studi di Torino" (lamieri@econ.unito.it). Piazza Arbarello 8, 10122 Torino Italy 


\section{Contents}

1 Introduction 4

2 Firm and innovation, knowledge and networks 4

2.1 Innovation, firm and economic growth: the schumpeterian heritage ........................ 5

2.2 Knowledge, interaction and the innovative process . . . . . . 6

3 Firm and relationships: small worlds and social networks $\quad 7$

3.1 Social networks: why the world is a "small" world? . . . . . . 7

3.2 Graph theory . . . . . . . . . . . . . . . 8

3.3 Small worlds: networks between order and chaos . . . . . . . . 9

4 An agent based model on innovation creation and diffusion 11

4.1 Simulation techniques for social sciences and economics . . . . 11

4.2 The consumer side . . . . . . . . . . . . . . . . . . 14

4.2.1 Consumer with heterogeneous preferences . . . . . . 14

4.2.2 Consumer and bounded rationality . . . . . . . . . . . 14

4.3 The firm's side . . . . . . . . . . . . . . . . . . 15

4.3.1 The technology is embedded in the products . . . . . 15

4.3.2 The financial aspects, a basic accounting framework for the firm . . . . . . . . . . . . . 15

4.3.3 Internal research and imitation: the innovations strategies . . . . . . . . . . . . . . 16

4.4 Model's dynamic . . . . . . . . . . . . . . . . . . . 16

4.4.1 The environment in which firms and consumers interact 16

4.4.2 Dynamics and interactions . . . . . . . . . . . 17

4.4 .3 The innovative process . . . . . . . . . . 18

5 The outcomes of the model $\quad 20$

5.1 The emergence of real business cycle in the simulated society . 21

5.2 Endogenous growth and monopoly . . . . . . . . . . . . 23

5.3 Research and imitation . . . . . . . . . . . . . . . . . 24

$\begin{array}{llr}6 & \text { Conclusions } & 27\end{array}$

$\begin{array}{ll}\text { References } & 29\end{array}$ 


\section{List of Figures}

1 One dimensional graph . . . . . . . . . . . . . . . . . . 9

2 Random graph ... . . . . . . . . . . . . . . . 9

3 From left to right: regular graph, Small Worlds graph and random graph (Watts, 1998) . . . . . . . . . . . . . 10

4 Simulated world with 100 consumer and 10 firms. The beta model connecting the firms has $\beta=0.1$ and degree $=2 \ldots . .17$

$5 \quad$ Network graph showing the relations among firms (red lines) and between customers and firms (gray lines) . . . . . . . 18

6 Imitation process . . . . . . . . . . . . . . . . . . . . . 19

7 Simulated world populated with 10 firms and 10 customers . . 21

8 Innovation dynamics . . . . . . . . . . . . . . . . . . 22

9 Average asset during the innovative process . . . . . . . . . 22

10 Aggregate asset statistics . . . . . . . . . . . . . . . . . 23

11 Continuous growth in asset moving average and effect on innovation . . . . . . . . . . . . . . . . . . . 24

12 Simulated world with 30 firm and 300 consumer: the competitive advantage of innovators . . . . . . . . . . . . 25

13 Simulated world with 30 firm and 300 consumer: the competitive advantage of imitators . . . . . . . . . . . . . 26

14 Growth due to knowledge spread . . . . . . . . . . . . 26

15 Imitation and innovation dynamics $\ldots \ldots \ldots \ldots . \ldots .27$ 


\section{Introduction}

In this paper ${ }^{1}$ we focus on both innovation and its relational properties. The simulation model presented investigates the dynamics of a population of firms and consumers interacting in a relational space, defined as a social network. Consumers are represented in the model in order to create a competitive environment pushing enterprises into innovative process (we refer to Schumpeter's definition): from interaction between consumers and firms innovation emerges as a relational good.

In section 2 a Schumpeterian definition of innovation is introduced and we present some insights on the role of knowledge in the innovative process of firms. Section 3 starts from a brief account on theoretical literature on social networks and graph theory. Social networks represent people and their relationships as networks, in which individuals are nodes and the relationships between them are ties. Moreover, graph theory is introduced in order to represent the network, which will be populated of both firms and consumers. Finally, the "small worlds" phenomenon is presented and implemented as an algorithm useful to define how relationships are formed. Finally a model drawn from literature is presented as an example.

The empirical section of the paper is introduced by a brief summary of simulation techniques in social science and economics, as a way to investigate complexity. The model is then presented, with a detailed description of its main features. In conclusion of the paper, some early results of simulation runs are presented and discussed.

\section{Firm and innovation, knowledge and net- works}

In this section we will provide a brief account of part of the theoretical debate on the role of innovation in the economic system. We will try to demonstrate, always from a theoretical point of view, why innovation is mainly a matter of relations and why we need to study networks of firms with our model.

\footnotetext{
${ }^{1}$ This paper is the result of a research made in cooperation by both the authors. The writing process has to be attributed to Marco Lamieri for sections 1, 2, 4 and to Daniele Ietri for sections 3,5 and 6
} 


\subsection{Innovation, firm and economic growth: the schum- peterian heritage}

The theories of economic development of "neo-classical" origin (or, as is often said, mainstream) have often considered technological innovation as a simple factor in a production function. Nevertheless Karl Marx in his "Zur Kritik der politischen Ökonomie" (1859) recognised in the technical progress the main reason for development. According to the German economist, in fact, competition forces entrepreneurs into the innovative process, which consists in the exploitation of what is made them available by science.

The role of innovation has been studied in-depth by Schumpeter (1934), who focused in particular on the role of the entrepreneur. Schumpeterian entrepreneur is characterised by exceptional qualities of force and sharpness and is the real author of the innovative process, as she is able to introduce innovations in the productive processes. Schumpeter's model formalises three steps of the innovative process. First, the invention, that applies to the outcome of research activity. Second the innovation itself, that occurs when invention is applied in the productive process. Third, imitation, which is defined as the attitude of other entrepreneurs imitating the original invention. In schumpeterian approach innovation is the re-organisation of productive factors in new combinations: the outcomes may concern the introduction of a new good or productive process, the opening of a new market, the discover of a new source of primary goods, the emergence of a new form of organisation of the firm ${ }^{2}$.

Innovation has characteristics of a "creative disruption" force, as it determines the rise of new activities and productive sectors to the detriment of old sectors and technologies. Moreover, the nature of the innovative process itself, as described by the Austrian economist, based on a perpetual dynamics of innovation - imitation - innovation, is cause of economic growth and mostly of the cyclical instability of the economic system. Innovation, in fact, is introduced by few early adopters and is diffused in the system by the imitators. In the aggregate dynamics of the model presented in this paper we will look for those cyclical behaviours: we will test if a sort of "bangwagon effect" as described by Shumpeter ${ }^{3}$ will emerge from the simulation.

\footnotetext{
${ }^{2}$ In the model proposed in this paper, which will be described in details in section 4 below, we focus on second and third step of the innovative process described. Even if we aware of the risks of excessive simplifications, we will describe only the case of an innovation concerning the introduction of a new good.

${ }^{3}$ After the first diffusion of the innovation, during which many new firms enter the new sectors and markets, follows a period of profit compression, which is then compensated by the introduction of new innovations. Shumpeter defines this dynamics as "bangwagon
} 
The schumpeterian approach inaugurated a long lasting theoretical debate: some recent works of the economists of the so called Austrian school are interesting for our theoretical framework. In particular the writings of Friedrich Hayek are fundamental for the attention they pay to the role of technical development and knowledge in the firm (Hayek, 1937). The main role of information and its transmission and diffusion dynamics is underlined by Hayek in a paper in which he explains how people keep specific pieces of knowledge, which are uneasily transferable to others.

\subsection{Knowledge, interaction and the innovative process}

The ways in which knowledge is produced and diffused play a fundamental role in the innovative process. Knowledge is often part of the know-how peculiar of a subject or an organisation. In order to define the framework for the simulation model presented below we will briefly present some definitions of knowledge and hypothesis on its diffusion dynamics.

Knowledge is very heterogeneous according to its nature and scope: it can be very abstract or applied, it can be used in a very technical or general way (both engineering and philosophy are forms of knowledge). According to the way it is diffused, Polanyi (1967) individuates two main kind of knowledge:

- codified knowledge: easily transferable and accessible to everyone who knows its specific language (or code), it can be sold or appropriated (i.e. through patents);

- tacit knowledge: is embedded in an organisation or community, it can be transferred only through personal relations and it can't be sold.

If we admit the existence of a such kind of knowledge we recognise that at least part of the knowledge involved in the innovative activities of firms has a tacit nature. As this kind of knowledge can be transferred through personal relations, innovation becomes a relational activity, in which relations and proximity between firms are needed. Relationships are then necessary in order to allow external knowledge to be used in the productive processes inside the firm, especially when tacit knowledge is involved. Imitation is possible, but many relationships are needed in order to internalise processes taken from the activities of other firms: this is why in our model firms would be able to imitate only the most diffused goods.

effect" 
More in general, innovation and the production of knowledge is a "localised" process: proximity matters, in relational and spatial terms ${ }^{4}$. That's the reason for the use of social networks in order to investigate innovation dynamics among a population of firms.

\section{Firm and relationships: small worlds and social networks}

In this section we introduce a more methodological issue: if we investigate relations, a topological definition of relationships is needed in order to build a simulation model. The necessary theoretical background is defined by three main concepts:

1. social networks, as a field of research that has studied in-deep the form and dynamics of the relationships among people. We will simply draw some concepts from literature and apply them on the relations between firms;

2. graph theory, as a way to represent topologically networks and define measures useful to get some of their main characteristics;

3. "Small Worlds" phenomenon, from which we derive a theoretical framework (and an algorithm) in order to simulate the creation and dynamics of relationships between agents.

\subsection{Social networks: why the world is a "small" world?}

Some features of social systems may be studied through the theoretical framework of physical systems. As an example, the notion of distance typical of physical systems can be adapted in order to suite the characteristics proper of relationships between people. The use of distance reveals also some critical difference between the two systems. A fundamental property of physical systems is that randomly chosen three points in the space and connecting them by segments we form the sides of a triangle. The sum of the length of the two sides is always greater than the measure of the third side. In social systems the concept of distance can be used to represent the strength of the relationships intertwined between people. The smaller is the segment connecting two agents, the strengthen will be their relationships. Social systems violate the

\footnotetext{
${ }^{4}$ Even if in the model presented below we do not explicitly define a geographical space, it will be implemented in the future developments of the simulation
} 
triangle property: a subject may in fact entertain very strong relationships (the segments connecting her acquaintances are very short) with two subjects that do not share any relation (there's no segment connecting them). An individual may belong to separate groups of acquaintances among which there's no relation: in this case the subject is the only connection between the two groups. We will call those distinguishable groups of acquaintances clusters: they are for examples the family, groups of friends, colleagues and other groups in which people participate for different purposes.

The "Small World" phenomenon is part of the studies on social networks. In a social network, individuals are represented, conceptually and graphically, as nodes of networks formed by the subjects themselves and the relationships connecting them.

\subsection{Graph theory}

In order to represent social networks and the Small Worlds phenomenon we should briefly introduce some concepts of graph theory, following Watts (1998). We will define graph any set of points (vertexes or nodes) tied by lines (links or edges). The number of points defines the order of the graph, while the number of lines defines its dimension. Some properties of graphs useful for this work are:

- the characteristic path length $\mathrm{L}$, defined as the median of the means of the shortest path lengths connecting each node to all others nodes: randomly chosen two subjects in the social network, we can expect being $\mathrm{L}$ the length of the shortest path between them;

- the cohesion $\mathrm{d}$, the probability that nodes linked to any defined node would be connected to each other . This property is also defined clustering coefficient and is the ratio between the number of edges in the neighbourhood of a node and the total number of theoretically possible edges in that neighbourhood. It is a proxy of the tendency for a graph of forming clusters, sub-groups of nodes.

Thanks to the analysis of graphs itis possible to study the relations between local and global level of the network. At the local level the graph has nodes connected in a way determined only by the neighbouring conditions, with each node linked to its neighbouring nodes: in Figure 1 this condition is graphically presented.

If the edges are randomly determinate we obtain a random graph (global graph), represented in one-dimension in Figure 2. 


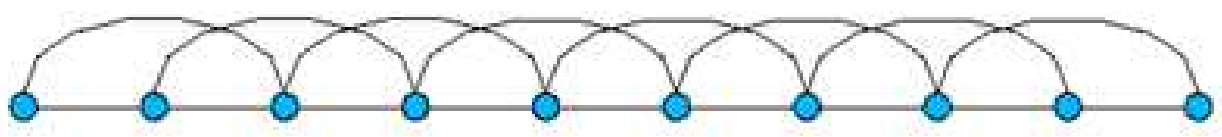

Figure 1: One dimensional graph

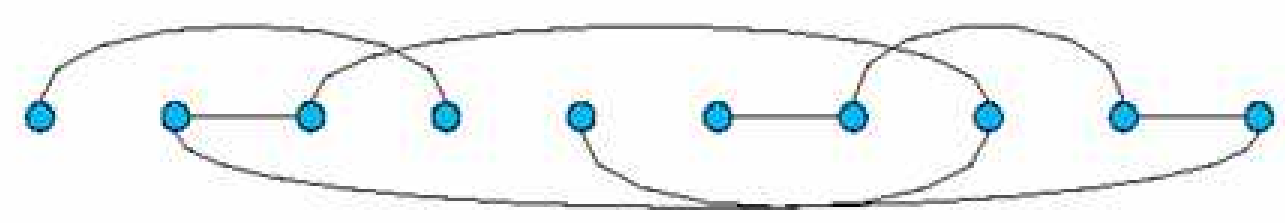

Figure 2: Random graph

If nodes represent the agents and graph formed by nodes and ties represent social networks, then we justify the use of terms such as "local" and "global". In a structure such as the one of the regular graph, each node (agent) is able to interact only with the $\mathrm{k}$ agents forming its neighbourhood: the space determines the possibility to interact of each agent that may operate only in a local context. The agents interacting in a random graph may establish relationships apart from the spatial structure of their neighbourhood, acting in a pure global scale.

\subsection{Small worlds: networks between order and chaos}

Defining from end to end the possible structure of graphs, on one end onedimensional structures perfectly correlated and on the other multi-dimensional structures perfectly un-correlated, we can explore (Watts, 1998) the intermediate configuration, looking for "small worlds" graphs, characterised by high levels of both accessibility and cohesion.

We take into consideration, apart from the sociological meaning of the network, a circular structure that, varying a parameter $\beta$ gradually changes from a determined graph into a completely random graph (Figure 3). Watts (1998) presents some results with respect to characteristic path length and clustering coefficient measures. When $\beta$ is very small $(\beta=0.01)$ the characteristic path length gets immediately lower, while the clustering coefficient lowers only for higher levels of $\beta$ ( $\beta=0.1)$. In the interval $(0.01 \leq \beta \leq 0.1)$ the graph presents both cohesion and accessibility.

The accessibility of the network increases while the $\beta$ parameter increases and is due to the way in which ties are recalculated. If the number $\mathrm{k}$ of 

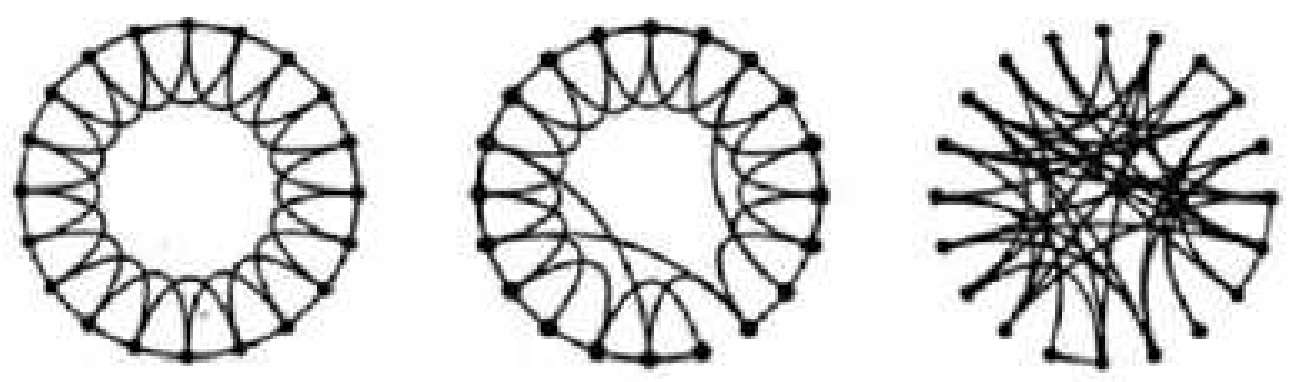

Figure 3: From left to right: regular graph, Small Worlds graph and random graph (Watts, 1998)

connections is fixed, while $\beta$ increases the dispersion of ties grows.

For low levels of $\beta$, new ties connect two nodes which already share a neighbourhood with a third one: a triangle is formed and the path within them is scarcely reduced. We will define this feature as a "shortcut". While $\beta$ grows new ties will instead connect very distant nodes that do not share their neighbourhoods. In this way the new links created will connect groups of agents, reducing the characteristic path length of the network.

Parts of the graph and groups of nodes which otherwise would be separated are connected thanks to this feature. A few shortcuts are needed in order to obtain a sensible reduction of the characteristic path length: in fact, it lowers immediately for low levels of the $\beta$ parameter.

The implementation of graph theory in order to explain the innovation diffusion is a fertile ground of study; for example Cowan and Jonard (1999) focus on knowledge diffusion through face-to-face relationship and on the relationships developed on networks structures. It is assumed that subjects are part of a network and detain various kinds and amounts of knowledge. For each step one of the edges of the network is randomly chosen and the subjects connected by that edge compare their knowledge. If for example one of them detains more knowledge of the kind $a$ and the other of the kind $b$ they get involved in an exchange of knowledge. The parameter in the model is the probability $\mathrm{p}$ that a link is destroyed and re-wired. For $p$ varies, the measures of characteristic path length and cohesion are calculated: for $p$ included between 0.005 and 0.01 , the model shows high cohesion and low characteristic path length. Cowan and Jonard measure the medium level of knowledge detained by each subject: the level is particularly high in the same interval $(0.005 \leq p \leq 0.01)$. We may say that cohesion and path length act in opposite directions: cohesion is responsible for a quick diffusion among neighbours (at local level) while path length shows how information (or innovations) is able to reach and diffuse far from the point in which have 
been originated.

\section{An agent based model on innovation cre- ation and diffusion}

The analysis of innovation diffusion within a social network present some analytical difficulties, due to the emergence of global properties not predictable on the basis of local interaction. Computer simulations seem to be suitable in studying those phenomena, which undoubtedly present elements of complexity.

\subsection{Simulation techniques for social sciences and eco- nomics}

According to Langton (1992) one of the fundamental aspects in the analysis of complex phenomena is the distinction between linear and non-linear systems.

Dealing with linear systems the behaviour of the whole system exactly corresponds with the sum of its constituting parts. Obeying to a principle called by Langton "superposition principle", linear systems may be studied with a reductionistic method. The system is divided into simpler parts, whose understanding is enough in order to understand how the system works as a whole. In this case it is possible to say that the system is "just" complicated.

Non linear systems do not respect the superposition principle: even if the observer has understood how each part works, it won't be possible for him to understand the system as a whole. This kind of system is complex. The fundamental difference between a complicated and a complex system is the importance of connections between parts, which would fail if parts are considered separately.

Dealing with a system presenting this kind of features, the research method has to be deeply revised in comparison with the analytical tradition. Instead of starting from the system as a whole in order to decompose it and understand its parts, it would be more opportune to start from its constitutive parts.

Complex systems show other properties, such as emergence. If we define each part's internal and interaction rules, the outcome of the system as a whole may be different from the bare sum of its parts. Coherent behaviours not defined a priori may spontaneously come out from the aggregate dynamics. A property is defined emergent if it clearly results from the behaviour of the system, even if it has not been defined before in its parts' rules. 
The need to study complex system "bottom-up" lead to the diffusion of agent based simulations methods, where agents are comparable to what has since defined parts. For a thorough analysis of agent based simulations in social science, economics in particular, we refer to Gilbert and Terna (2000) and to Tesfatsion $(2002)^{5}$.

According to Parisi (2001, our translation):

Simulations carry out a synthesis of reality, where synthesis means starting from elements to study how they interact and the emergent behaviour. A simulation stems from a group of components of a system and, through the elaborations of a computer, shows how the interactions between elements yield to the overall system with its specific properties. Simulations are based on the assumption that reality can't be explored just through the analyses of its components, but is necessary to rebuild it beginning from its constructing components.

Simulation models are methods used to comprehend reality halfway between models expressed by language and mathematical symbols. Mathematical models appear to be too much rigid, as they don't allow to adequately highlight the behaviour of parts composing the model without overloading them exceedingly complex rules. Verbal models are suitable to be more flexible, but are unfit for providing quantifiable results: even if they overtake the rigidity of mathematical models, they are surely less rigorous.

A simulation model does not use words or mathematical symbols: the phenomenon is described with a formal language suited for the computer. The computer does calculation and provides the results for the experiment. In this way the computer works like a sort of virtual laboratory through which the scientist can verify his hypotheses, manipulates variables and constraints of the model and afterwards observes the outcome on the screen.

The empirical part of the paper is an agent based simulation model that tries to describe the economic law that lead the enterprise into an innovative process. The model describes how the innovations are created and the dynamics of knowledge spreading to other enterprises via imitation ${ }^{6}$.

From a technical point of view we use Swarm (http://www.swarm.org) as agent based simulation framework to develop the model and implement it in Java (http://java.sun.com). Swarm has been chosen because of its diffusion

\footnotetext{
${ }^{5}$ For a discussion about simulation methods and their applications on social system see Parisi (2001).

${ }^{6}$ The software including source code and the instruction on how to use it are available from http://innovation-abm.sourceforge.net
} 
and the presence of a strong community of users; we believe is very important to create a model easily understandable by other scientist and Swarm is somehow a base framework. In order to visualize the simulated world of firms and customers interconnected, the library Jung - Java Universal Network/Graph Framework (http://jung.sourceforge.net/) has been used.

The simulation represents the economic world using two different populations of agents:

1. firms

2. consumers

The focus of the model is the enterprise which is the potential innovator: consumers define a competitive environment for the enterprises in order to push them into an innovative process.

In the definition of innovation (or better "innovative process") we refer to the field of study leaded by Schumpeter. We consider the innovation as a relational good, and we will show that the innovation's value emerges from the interaction between firms and consumers. We refer to innovation as the process of discovering something new; we refer also to the imitation process that is crucial in order to spread knowledge in the economy trough a social network.

We focus especially on two theoretical concepts.

1. the role played by the tacit knowledge in the innovative process. This kind of knowledge is transferable only by face-to-face relationship via a non codified transmission. Tacit knowledge is idiosyncratic of a specific time, place and group of people.

2. social relations are expensive in term of time and effort both for firms and for consumers; in order to draw a social network we try to justify all the relations in terms of competitive advantage or higher knowledge obtained by the members.

The goal of the simulation is to build a model useful to understand the innovation process from two different points of view.

1. From a micro perspective we investigate:

(a) why the firms decide to innovate;

(b) how the innovation process affects the enterprise's activity;

(c) how innovation spreads via imitation. 
2. From an aggregate perspective we look for emergent phenomena coming from the interactions of the simple agents described in the model, the firms, and we investigate:

(a) effects of innovations and imitations on the economy as a whole;

(b) business cycles dynamic of the simulated economy;

(c) technological progress dynamic at a macro level: is it constant or does it shows, jumps, radical innovations followed by incremental ones?

\subsection{The consumer side}

Consumers define the environment for the enterprises but they are not object of analysis. Consumers have heterogeneous preferences and bounded rationality.

\subsubsection{Consumer with heterogeneous preferences}

The preferences of the consumers are defined by a numeric array, each value of which is a "feature" of the product that the consumer would like to have. Every consumer has its own preferences randomly generated at the beginning of the experiment using an array of integers with a length defined by the initPreferenceLength parameter. The domain of each value is defined by the parameter preferenceDomain. For instance a consumer preference could be defined as a 3 digit string using an alphabet of 12 possible values for each digit.

$$
[11 ; 0 ; 3]
$$

Every consumer looks for a product as much similar as possible to its preference.

\subsubsection{Consumer and bounded rationality}

For a theoretical perspective on bounded rationality in economics we refer to Ludwig von Mises (1949) and Camerer (2004). The bounded rationality of the consumer is modelled using a social network; the consumers are vertex of a graph and are connected with the enterprises via undirected edges. If a consumer is connected to a firm with a edge it means that the consumer knows that firm and, most important, she knows the product offered.

The edges between consumers and enterprises are dynamics and are modified during the simulation as described in section 4.4. The consumer buys 
one product at every tick and she chooses the one closest to her preferences among the known products.

\subsection{The firm's side}

\subsubsection{The technology is embedded in the products}

Each firm produces one product, represented by a numeric array of a defined length. Each value of the array represents a technological feature of the product: in this way each product is potentially different from each other product. The parameter initProductsLength defines the initial length of the products for all the firms. When the simulation starts each firm is characterized by a product defined by many features chosen randomly within a domain defined by the parameter featureDomain.

For instance a product could be defined as a 4 digit string using an alphabet of 12 possible values for each digit; in this case a possible product would be:

$$
[1 ; 11 ; 0 ; 3]
$$

\subsubsection{The financial aspects, a basic accounting framework for the firm}

The firm has a basic accounting capability; the firm has an initial asset defined by the parameter initialFirmAsset and at every tick of the simulated time the firm faces costs and revenues.

Costs are divided in:

1. fixed costs, defined by the parameter fixedCost; they are subtracted from the firm's asset at every tick. All the firms pay the same fixed costs;

2. variable costs; they are due to the innovative process and are paid only if the firm decides to innovate. There are two kinds of variable costs:

(a) cost for internal research: in case the firm decides to innovate investing in research a parametric defined cost researchCost is charged. This is generally an high cost;

(b) cost for imitation: in case the firm decides to imitate another firm's product a parametric defined cost imitation Cost is charged. This is generally a low cost. 
The revenue comes only from selling activity: when a firm sells a product, it earns a predefined amount of money. The revenue depends only on the number of products sold.

\subsubsection{Internal research and imitation: the innovations strategies}

The firm has also an innovation strategy; firms can have a vocation to imitate or to be engaged in internal research. Each firm's innovation strategy is decided randomly (with 0.5 probability from a normal distribution) at the beginning of the simulation. At every tick the firm decides its innovative strategy according to its vocation; an "imitation strategy" firm will always innovate by imitation and a "research strategy" firm will always use internal research to innovate.

\subsection{Model's dynamic}

\subsubsection{The environment in which firms and consumers interact}

Firms and consumers are connected each other within a social network. The network is defined topologically as a graph with vertex (firms and consumers) and edges (their relations). This is the relational space of firms and consumers.

Firms are connected among them using a $\beta$ small world graph as defined by (Watts, 1998) and their edges are static. This kind of model has been chosen for its statistical property (it shows "Small Worlds" properties) as described in chapter 3 .

The connection between firms and customer is dynamic, evolves over time, and is defined randomly with a given probability. The formalization of the ties between firms and consumers is randomly generated at the beginning of the simulation and refreshed every $n$ tick where $n$ is defined by the parameter edgeUpdateFrequency. At every refresh the following algorithm is performed:

1. for every consumer the existing edges are destroyed with a probability $p 1$ defined by the parameter edgeDistructionProb;

2. for every consumer, excluding the existing edges not destroyed in phase 1 , new edges are created using a probability $p 2$ and testing it for every possible link between a customer and all the existing firms; $p 2$ is defined by the parameter edgeCreationProb.

Figure 4 shows a configuration with 100 consumer and 10 firms. The $\beta$ model connecting the firms has beta 0.1 and the graph degree is 2 ; the 
probability of edge creation between consumer and firms is 0.2 . To visualise the graph the Fruchterman-Reingold algorithm has been used.

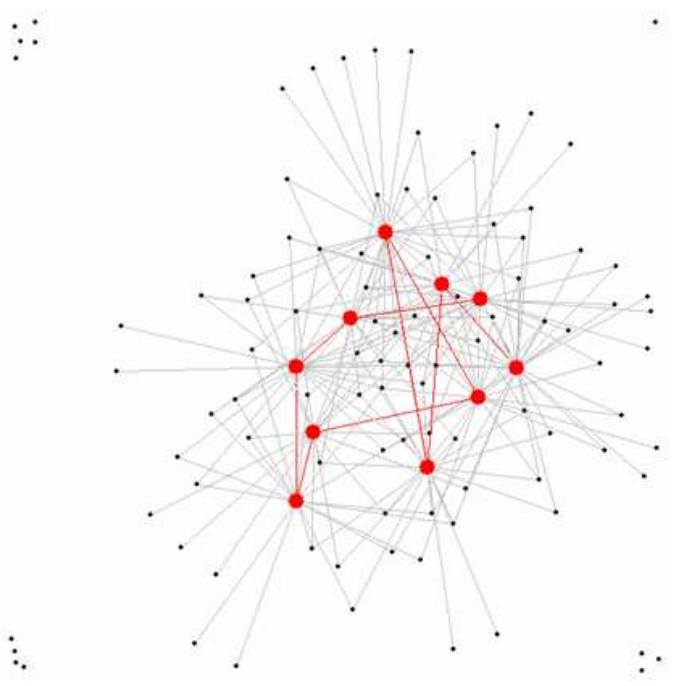

Figure 4: Simulated world with 100 consumer and 10 firms. The beta model connecting the firms has $\beta=0.1$ and degree $=2$.

\subsubsection{Dynamics and interactions}

Each consumer looks for the best product to buy selecting among the firms she knows, then chooses the product that better fits its preference. At every simulation's tick each consumer buys one product ad generates revenues for the selling enterprise. In order to choose the better product, the consumer compares her preferences with all the products she knows and chooses the one that has more common values. For example consider a consumer preference and 3 products:

$$
\begin{gathered}
\text { Consumer preferences }[1 ; 3 ; 7] \\
\text { Product } 1[5 ; 9 ; \underline{1} ; 4] \Rightarrow 1 \text { match } \\
\text { Product } 2[6 ; 9 ; \underline{7} ; 5 ; \underline{7} ; \underline{1} ; 2 ; \underline{3}] \Rightarrow 4 \text { match } \\
\text { Product } 3[2 ; 11 ; 4 ; 8 ; \underline{3} ; \underline{1} ; 10 ; 5] \Rightarrow 2 \text { match }
\end{gathered}
$$

Product 2 will be chosen and bought by the consumer. It is easy to see that longer features strings have higher probability to be chosen; if we remember that, starting from an initial length equal for all the products, 


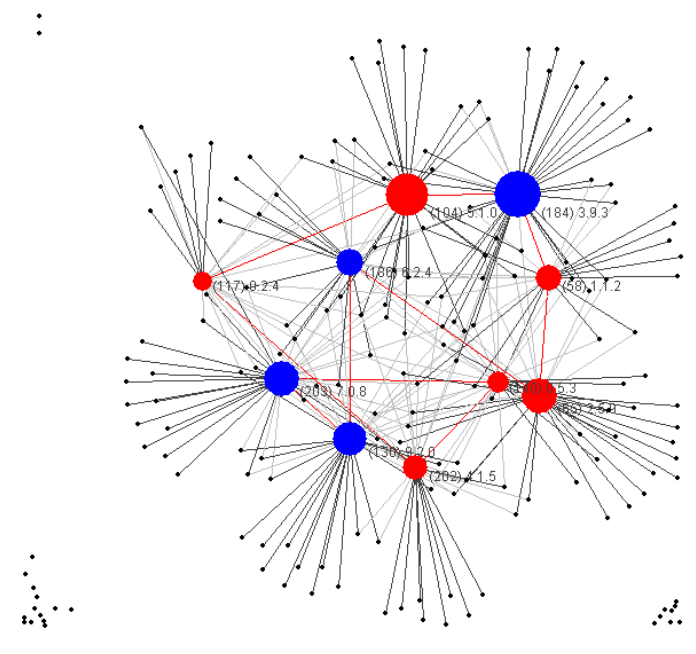

Figure 5: Network graph showing the relations among firms (red lines) and between customers and firms (gray lines)

firms can add features by innovation, it is easy to understand how we model the competitive advantage given by the innovation.

The network graph shows relations among firms and between firms and customers. The firms are connected among them by red edges. The firm's vertex colour depends on the strategy used by each firm to innovate. Blue vertexes are imitators and red vertexes are innovators. When a firm innovates, it becomes green.

The numbers displayed next to firm's vertexes show the firm's identification number, in bracket, and the feature of its product.

The customers are gray vertex and they are connected to the firms by gray edges. Each customer knows only the firms which is connected to by an edge and at every tick decides to buy from one of the firms she knows. The link connecting the consumer with the firm she buys from changes its colour into dark gray, while all the other edges remain light gray.

\subsubsection{The innovative process}

Innovation, when occurs, adds a value to the product features string, according to the strategy used by the firm to innovate. We have two possible strategies:

1. internal research: if the enterprise decides to innovate through internal research pays the amount defined by the parameter internalResearchCost. The research activity will then give a result with the probability 
defined by the parameter discoveryProb. We have two possible scenarios:

(a) internal research gives no results: the firm pays the cost for research and has no benefit at all;

(b) internal research is successful: the firm pays the cost for research and then adds a new feature (number) to its product (array of values). The feature is randomly chosen within the domain.

2. imitation: if the enterprise decides to imitate, it pays the cost of imitation defined by the parameter imitationCost. In order to imitate, the firm looks to the products of the other firms it is connected to in the network. The firm then chooses the feature most diffused among its neighbours and adds it to its product's feature list. If two or more features have the same diffusion within the neighbours one is chosen randomly. As an example the following graph shows the firm number 14 (the green one) imitating the feature " 2 " from its neighbours (firms number 3 and 16).

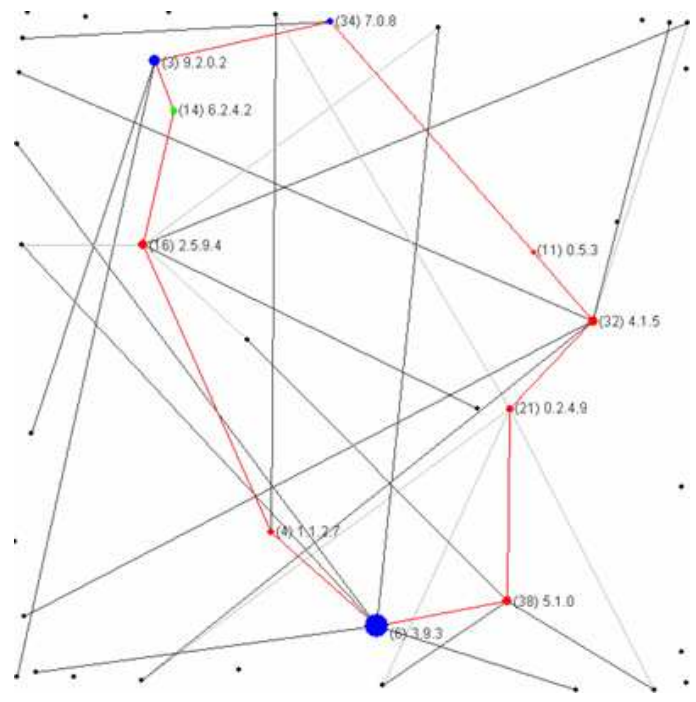

Figure 6: Imitation process

Firm $3[9 ; \underline{2} ; 0 ; \underline{2}]$

Firm $16[\underline{2} ; 5 ; 9 ; 4]$ 
Firm $14[6 ; 2 ; 4 ; \underline{2}]$

Usually imitation is cheaper than internal research and is more efficient in the model, as it outcomes with a certain result while research is only probable.

Innovating, and then adding features to the products, gives to the innovative enterprise a competitive advantage: its product is more likely to match consumer's preferences and then will probably sell more. The firms that perform internal research, discover something new that can provide competitive advantage regard to other firms in satisfying customers' preferences.

The main issue is: when the enterprise decides to innovate and why? According to Schumpeter we believe it happens for a competitive reason; the firm looks for higher profit or better performance. In accord to this theory we define two possible rules that lead a firm to innovate:

1. no past sell: a firm innovates if in the last tick it sells nothing. If the firm sells nothing it is probable that its product is not satisfactory for the consumers;

2. low market share: a firm innovates if its asset is lower than the $70 \%$ of the average asset of the neighbouring firms. If the firm connected have higher assets it means they sell more and that the market gives the opportunity to increase the revenue by finding a better product. An obvious conclusion is that innovation will lead to better economic performance.

It is important to remember that the kind of innovation (imitation or internal research) used by each firm is decided by its vocational innovative strategy as described before.

\section{The outcomes of the model}

From an aggregate point of view many aspects could be analysed using the data generated by the described model. In this section we would like to focus on:

1. real business cycle in the simulated society;

2. technological progress trend within the simulated society;

3. innovation diffusion process. 


\subsection{The emergence of real business cycle in the simu- lated society}

As a fist example we outline how a very simple configuration of the model provides a synthesis of the overall features. We see that firms are involved into the innovative process, relationships between enterprises and customers are activated; moreover, this experiment shows how an aggregate cyclical behaviour emerges.

In the example shown in Figure 7, the network is populated with 30 customers and 10 enterprises. Fixed and research costs as well as sell revenues are very low (respectively 1, 3 and 1) and the simplest innovation decision criterion is implemented (firms innovate if in the last time step no sell occurred) 7 .

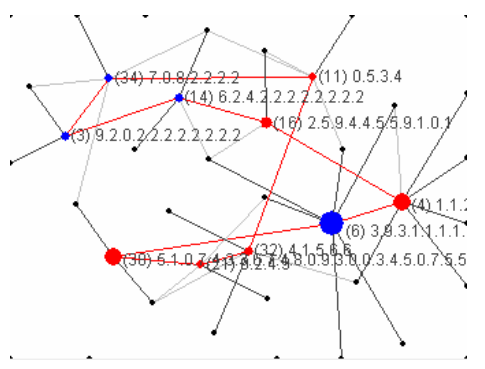

Figure 7: Simulated world populated with 10 firms and 10 customers

First of all, innovation occurs: enterprises do innovate (numbers/features are added to the simple string describing their products), via internal research and imitation. This is clearly visible through the dynamics of products array length shown on the graph and through the value of innovation moving average (Figure 8. The effect of relationships on imitation is clear, as the most diffuse feature will be reproduced into the imitating neighbourhood.

The technological progress is represented by the average length of the products features. The graph on the left shows the maximum, minimum and average length of the features; the graph on the right is a measure of efficiency of the technology in economical terms. The efficiency ratio for the firm $i$ is computed as:

\footnotetext{
${ }^{7}$ The parameters used are: firm Number 10, customerNumber 30, initialFirmAsset 10, sellRevenue 1, fixedCost 3, varCostCoefficient 0, researchCost 3, imitationCost 1, discoveryProb 0.8, graphBeta 0.1, graphDegree 2, edgeUpdateFrequency 1, edgeCreationProb 0.02, edgeDistructionProb 0.2, preferenceDomain 10, featureDomain 10, initPreferenceLength 3, initProductsLength 3, preferencesMatchCriterion 0, innovationDecisionCriterion 0 , imitationStrategy 0.
} 


$$
E R_{i}=\frac{\text { sellsNumber }_{i}}{\text { product Length }_{i}}
$$

The trend is upward sloping and it shows how the simulated society is innovating constantly over time.
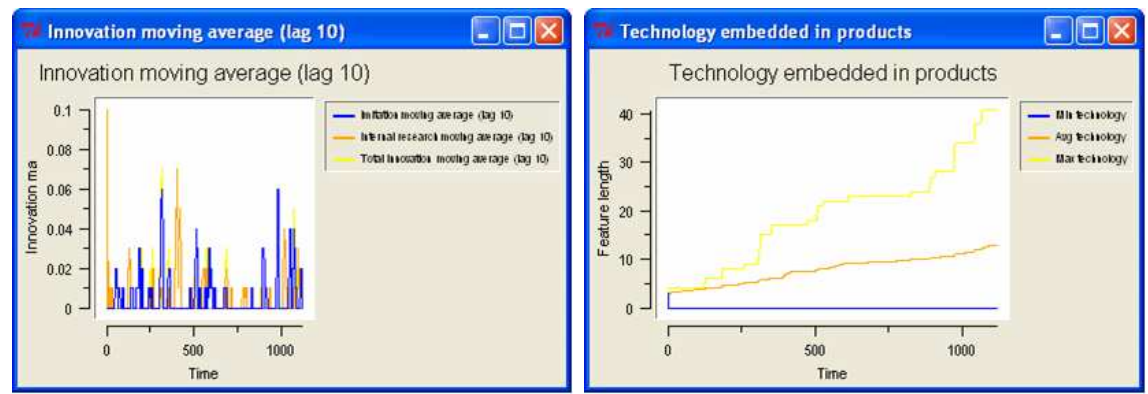

Figure 8: Innovation dynamics

It is easy to verify that innovation gives to the firm a competitive power, that is expressed in term of a higher asset. The graph shows the average asset of each firm when it decides to innovate and the average asset when the same firm is not innovative.

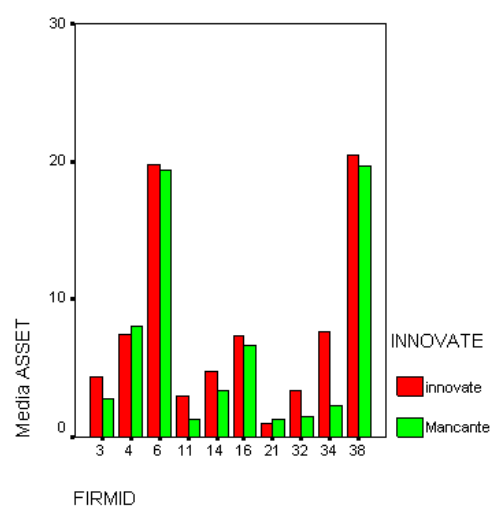

Figure 9: Average asset during the innovative process

Consumers, in this simple example, effectuate their buying decision comparing the products offered by the different firms which they are connected with. Links are activated at each time step and some firms have notable growth of their asset (the size of the circles, which describe firms on the graph, grows). 
Figure 10 shows a growth path generated by the model described. The graph on the left shows the maximum, minimum and average asset owned by the firms; the right one displays a moving average with lag 10 tick of the asset of the firms.

There are clear cycles, that could be conduced to the real business cycle theory: the expansion phases are followed by contractions. It is possible to identify big shifts and small adjustments alternated. Also the gross domestic product of the simulated economy confirms this outcome.

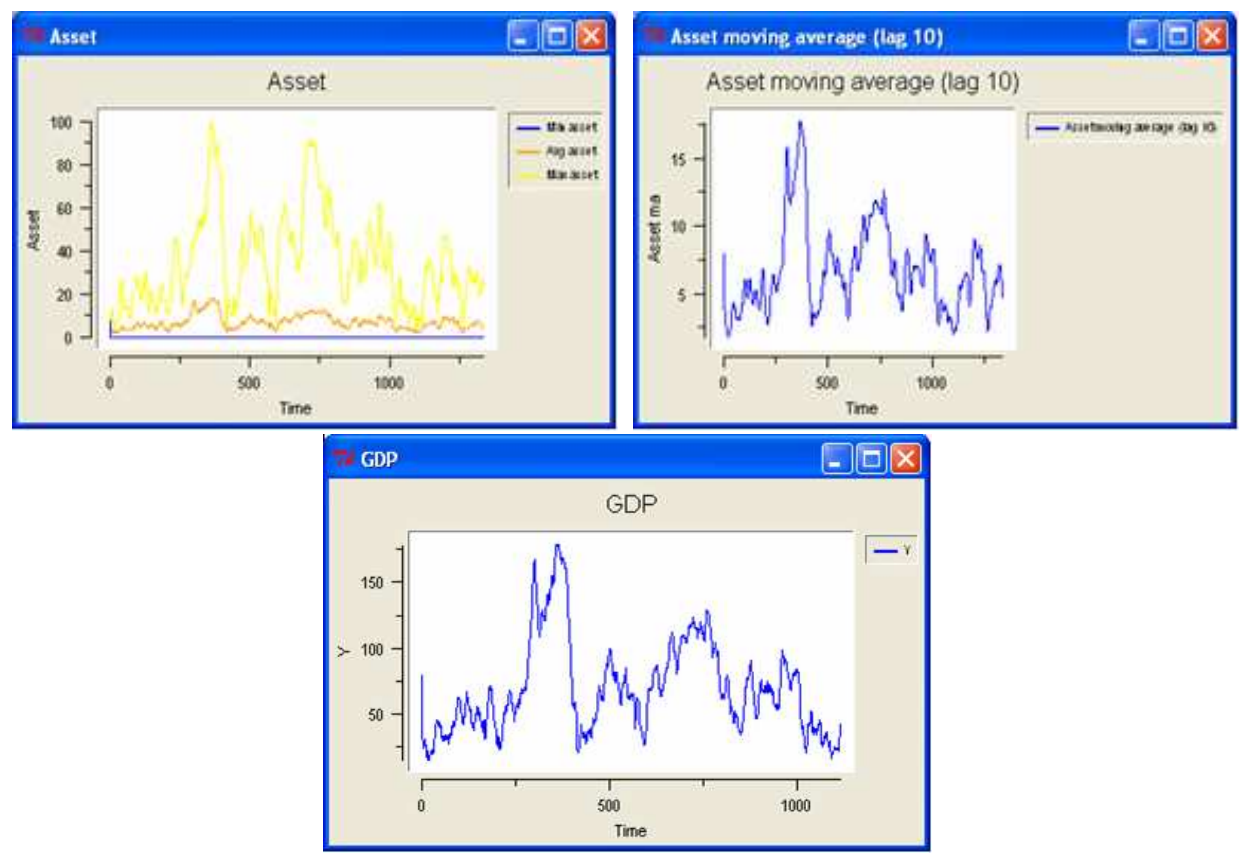

Figure 10: Aggregate asset statistics

\subsection{Endogenous growth and monopoly}

This second configuration ${ }^{8}$ tries to figure out the impact on the simulated economy as a whole of the innovations made by the enterprises. The effect of the innovations, within this framework, is continuous growth.

\footnotetext{
${ }^{8}$ The parameters used are: firm Number 30, customerNumber 300, initialFirmAsset 10, sellRevenue 1, fixedCost 30, varCostCoefficient 0, researchCost 80, imitationCost 10, discoveryProb 0.8, graphBeta 0.1, graphDegree 4, edgeUpdateFrequency 1, edgeCreationProb 0.02, edgeDistructionProb 0.2, preferenceDomain 10, featureDomain 10, initPreferenceLength 3, initProductsLength 3, preferencesMatchCriterion 0, innovationDecisionCriterion 0, imitationStrategy 0.
} 
The growth of the economy is not created exogenously by a parameter but it is endogenous and comes from the firm's interactions. Consumers in the model do always buy firms' products: they only decide which product to buy among the enterprises which are connected to. In this experiment we see how many consumers provide the opportunity for the firms to grow their assets through sells and we will investigate if monopolistic behaviour is coherent with the model.

The number of consumers is much higher than the number of firms (30 firms and 300 consumers): the model shows (ceteris paribus) a behaviour coherent with its construction, a condition of continuous growth, in firms' asset terms and for aggregate mean assets too (Figure 11).
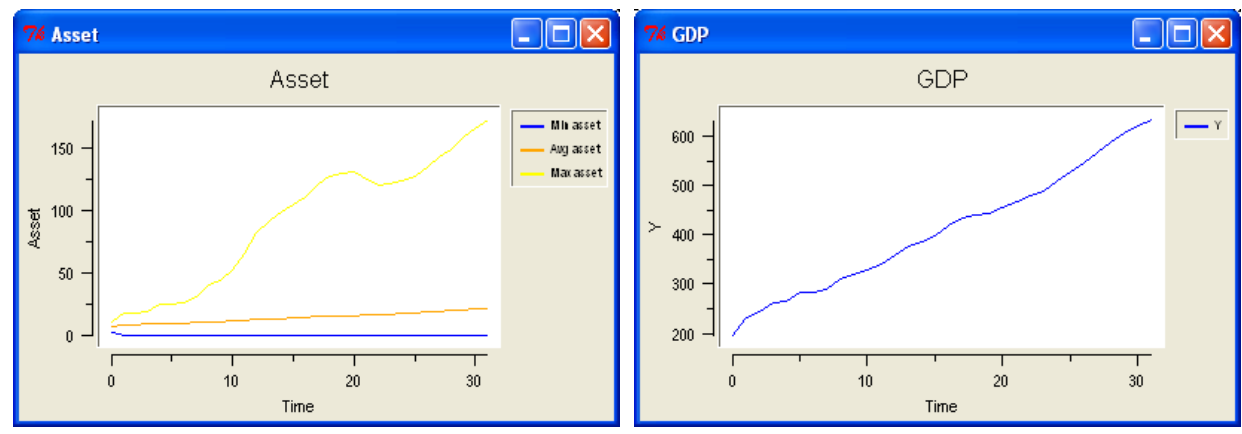

Figure 11: Continuous growth in asset moving average and effect on innovation

The growth is not homogeneous among firms (Figure 12); some of them, the most innovative ones, grow faster than others; this difference is due to the competitive advantage given by internal research, imitation or links with consumers, that let them gain a major share of the market.

\subsection{Research and imitation}

An important feature of the model, in the theoretical framework we outlined at the beginning of this paper, is the relational basis of the decision of firms to commit into the innovative process. In this simulation a firm decides for innovation if its asset is lower than the 70 per cent of the average asset of its neighbourhood. In the simulation ${ }^{9}$ the effects of this criterion are fully

\footnotetext{
${ }^{9}$ The parameters used are: firmNumber 30, customerNumber 300, initialFirmAsset 10, sellRevenue 1, fixedCost 20, varCostCoefficient 0, researchCost 80, imitationCost 10, discoveryProb 0.8, graphBeta 0.1, graphDegree 4, edgeUpdateFrequency 1, edgeCreationProb 0.02, edgeDistructionProb 0.2, preferenceDomain 10, featureDomain 10, initPreferenceLength 3, initProductsLength 3, preferencesMatchCriterion 0, innovationDecisionCrite-
} 


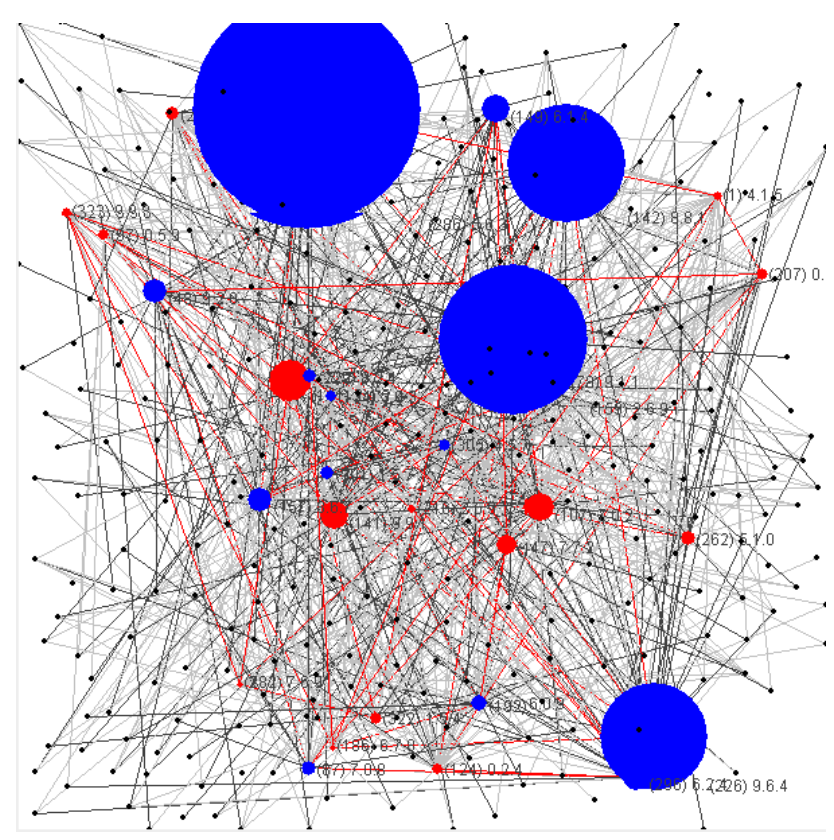

Figure 12: Simulated world with 30 firm and 300 consumer: the competitive advantage of innovators

evident in Figure 13: first of all, when just four firms out of 30 demonstrate very high levels of their assets and all of them are imitators, neighbouring firms do regularly choose the innovative process (via imitation or internal research).

The aggregate behaviour is similar to the imitation process described in Schumpeter's works: when a firm achieves a competitive advantage in the market, reaching a sort of monopolistic position, other entrepreneurs are aware of profit opportunities and get into the innovation-imitation process. Even if the profit of the quasi-monopolistic enterprise do not lower sensibly in the simulation, the aggregate asset of firms grows, as imitators' assets do as shown in Figure 14.

The smaller firms try to fill the competitive gap by innovating regularly, in this way, if they are imitative for vocation, contribute to the spread of the brand new technology created. The dynamics of the innovation is shown in the Figure 15.

rion 1, imitationStrategy 0. 


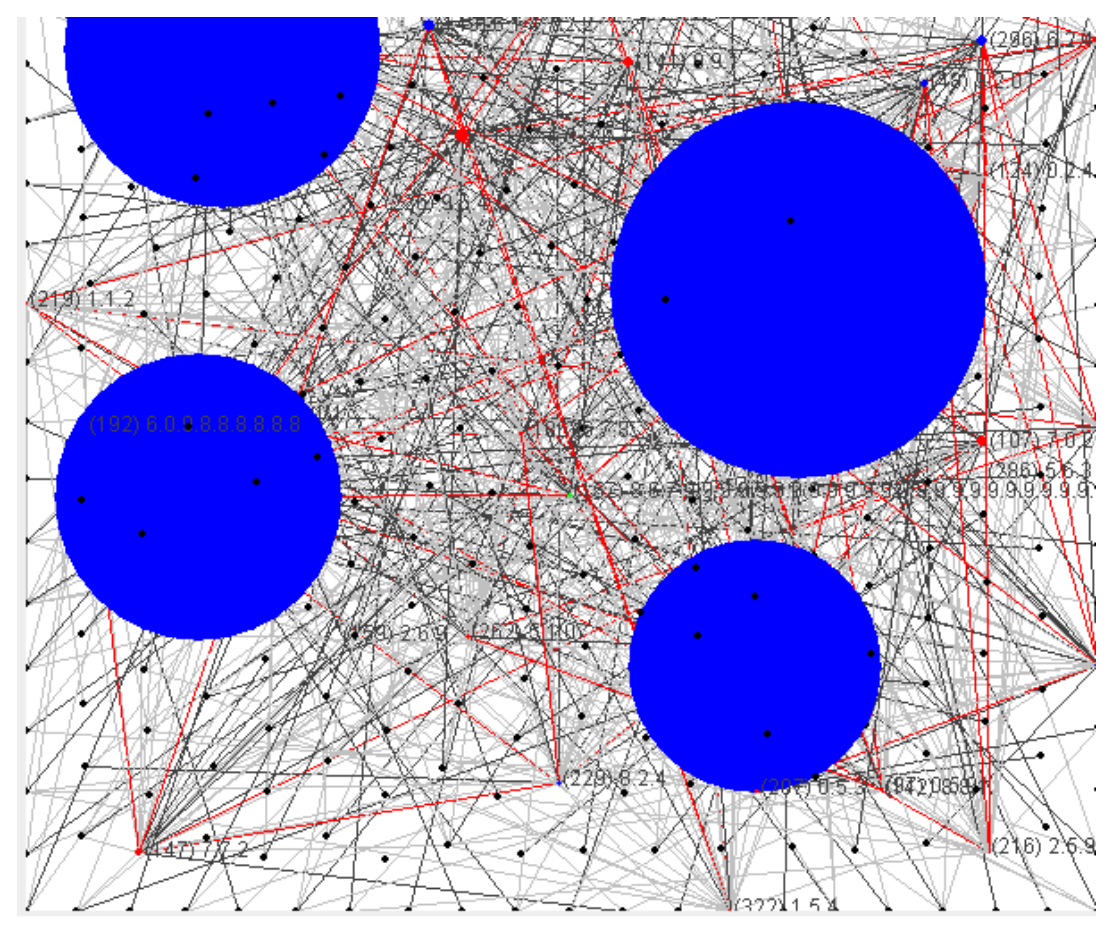

Figure 13: Simulated world with 30 firm and 300 consumer: the competitive advantage of imitators
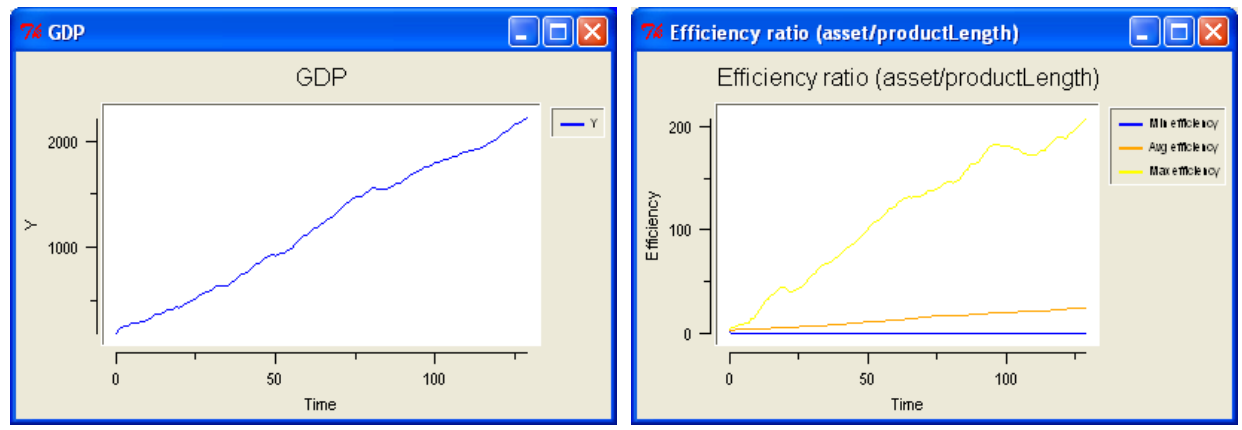

Figure 14: Growth due to knowledge spread 


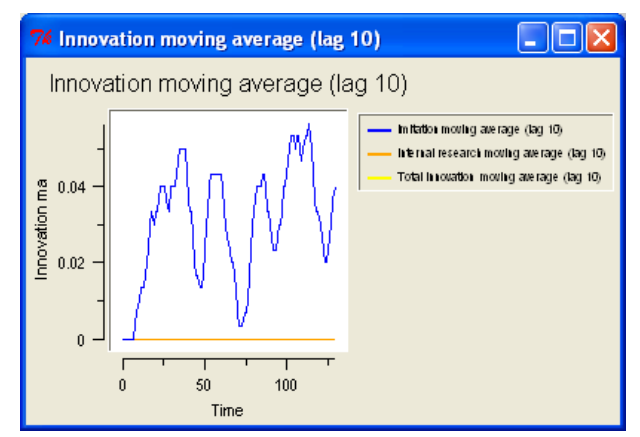

Figure 15: Imitation and innovation dynamics

\section{Conclusions}

In the previous section we described how the simulation model provided some useful insights for our understanding of firm innovation. Overall, the model shows how we can obtain coherent aggregate dynamics through the description of the only rules concerning parts, that in our example are firms and consumers. Computer simulation generates complex aggregate phenomena, such as innovation diffusion and business cycles: no description of the rules proper of this "macro" level was needed, as it emerged out from bottom-up dynamics.

We started our work from some theoretical deepening of economic literature on innovation; some insights on the nature and role of knowledge in entrepreneurial activity were useful to circumstantiate the subject of the simulation model. Relational aspects of innovation seemed to be more interesting and suitable to start the development of a simulation framework, which would be useful from both the point of view of economics and geography. We then explained how graphs and social network theory could be adequate in order to formally describe relationships between firms and consumers. We finally tried to implement coherently this theoretical framework into a simulation model.

The first results presented here seem to be encouraging as the main fundamental features that the model was expected to present did actually demonstrate to work correctly. First, a competitive market emerges: firms are forced into innovative processes by the push effect of consumers and we can say that the model presented has and endogenous growth force. The effect of relationships and the main properties of the network implemented in the model produce the expected outcome: innovation (in particular when imitation occurs) in firms is influenced by the conditions of their neighbourhoods. In general terms, "proximity matters". Moreover, some unexpected proper- 
ties emerge, such as the formation of cycles and, under certain conditions, monopolistic markets.

The encouraging results briefly summarised concur to imagine the provision for a follow-up of our work and future development of the model. First of all, more and more experiments should be done and new sets of variables have to be tested, in order get more validations of the model. Moreover the main issue for future developments will be the implementation of geographical space, beyond that relational one. In this future research, the use geographical information systems (GIS) will be fundamental, in particular with an integration between GIS and simulation software. The combined use of Swarm and some open source GIS will probably solve much of unsolved technical and theoretical issues. 


\section{References}

Cowan, R., \& Jonard, N. (1999). Network structure and the diffusion of knowledge. MERIT Research Memorandum(99-028).

Gilbert, N., \& Terna, P. (2000). How to build and use agent-based models in social science. Mind \& Society, 1, 57-72.

Hayek, F. A. (1937). Economics and knowledge. Economica(IV).

Langton, C. (1992, agosto). Vita artificiale. Sistemi Intelligenti, IV(2), $189-245$.

Parisi, D. (2001). Simulazioni. la realtà rifatta nel computer. Il Mulino.

Polanyi, M. (1967). The tacit dimension. Routledge and Kegan, London.

Schumpeter, J. (1934). The theory of economic development: An inquiry into profits, capital, credit, interest and the business cycle. Harvard University Press, Cambridge Mass.

Tesfatsion, L. (2002). Agent-based computational economics: Growing economies from the bottom up. ISU Economics Working Paper(1).

Watts, D. J. (1998). Small worlds: The dynamics of networks between order and randomness. Princeton Studies in Complexity. 